\title{
The Xpert MTB/RIF assay: A game-changer, but not infallible
}

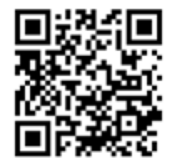

Tuberculosis (TB) is a major problem in South Africa and increasing varieties of drug-resistant strains add to the burden. The roll-out of the rapid molecular diagnostic test - the Xpert Mycobacterium tuberculosis/ rifampicin (MTB/RIF) assay (Cepheid, USA) - was a major game-changer in its ability to not only confirm MTB but also rifampicin resistance within a few hours.

However, two caveats have emerged in the recent literature that necessitate that we reconsider. These refer to the ability to detect all rifampicin-resistant strains and, since the test is so sensitive, the duration that the test remains positive after a diagnosis of TB.

The first investigators, in a study from Swaziland, used 24-loci mycobacterial interspersed repetitive unit - variable number tandem repeat (MIRU-VNTR) analysis and spoligotyping to perform classic genotypic analysis of MTB complex strains from their most recent national survey of tuberculosis drug resistance. ${ }^{[1]}$ They found that 38 of 125 multidrug-resistant strains (30\%) carried the rpoB I491F mutation, which confers resistance to rifampicin. This mutation, which was previously reported with low frequency in clinical isolates from other parts of the world, is not detected by the Xpert MTB/RIF assay. Xpert is designed to identify rifampicin-resistance mutations in an 81-base pair region of rpoB (codons 426 to 452 ). Its inability to detect the rpoB I491F outbreak strain will result in underdiagnosis of rifampicin-resistant MTB and potentially lead to inadequate treatment.

Thus, if putatively drug-sensitive TB patients don't improve on treatment, think of adherence, suboptimal absorption, and so on, but also consider this: has the test (Xpert) missed multidrugresistant TB?

The second study examined the issue of persistence of Xpert positivity in patients who have previously been treated for TB. ${ }^{[2]}$ Many of their patients were noted to have false positive Xpert results when the tests were repeated many months later. There have been case reports where the test remains positive for up to 5 years after the incident case of TB. Since the test amplifies DNA, how quickly the threshold for detection is reached is dependent on the mycobacterial load and is reflected in the cycle thresholds (CT). The higher the CT, the greater the likelihood that there will be a false positive result. There has therefore been a call for guidelines on the use of Xpert in treatment-experienced subjects. For now, in patients who are being assessed for persistent or recurrent TB, culturing is essential as Xpert alone is inadequate for a confident diagnosis, and the latter should preferably not be performed.

\section{Elvis M Irusen}

Professor and Head, Division of Pulmonology, Department of Medicine, Stellenbosch University and Tygerberg Hospital, Cape Town, South Africa

\section{References}

1. Sanchez-Padilla E, Merker M, Beckert P, et al. Detection of drug-resistant tuberculosis by Xpert MTB/RIF in Swaziland. N Engl J Med 2015;372():1181-1182. [http://dx.doi. org/10.1056/NEJMc1413930]

2. Metcalfe JZ, Makumbirofa S, Makamure B, et al. Suboptimal specificity of Xpert MTB/RIF among treatment-experienced patients. Eur Respir J 2015;45(5):1504-1506. [http://dx.doi.org/10.1183/09031936.00214114]

S Afr Resp J 2015;21(2):34. DOI:10.7196/sarj.8443 